\title{
Aortic arch replacement using the branch-first and frozen elephant trunk techniques
}

\author{
Michelle Kim, George Matalanis \\ Department of Cardiac Surgery, Austin Health, Melbourne, Australia \\ Correspondence to: Michelle Kim. Department of Cardiac Surgery, Austin Health, Melbourne, Australia. Email: mkim1389@gmail.com.
}

Submitted Nov 26, 2019. Accepted for publication Feb 18, 2020.

doi: $10.21037 /$ acs.2020.03.01

View this article at: http://dx.doi.org/10.21037/acs.2020.03.01

\section{Clinical vignette}

A 71-year-old female presents with central chest pain and shortness of breath and further investigation reveals marked dilatation of the thoracic aorta. Computed tomography of the aorta (CTA) demonstrates a maximal diameter of the aortic root of $40 \mathrm{~mm}$, ascending aorta of $70 \mathrm{~mm}$, aortic arch of $57 \mathrm{~mm}$ and the descending thoracic aorta of $40 \mathrm{~mm}$. Transthoracic echocardiogram demonstrates a dilated left ventricle with severe global systolic dysfunction. The left ventricular ejection fraction is $33 \%$. There is moderate aortic regurgitation and moderate mitral regurgitation. Coronary angiography reveals no significant coronary disease.

Medical history includes hypertension, chronic bronchitis and current cigarette smoking. The patient is functionally independent with an exercise tolerance of up to $5 \mathrm{~km}$ walking on flat ground.

She is consented to undergo a first stage aortic valve replacement, mitral valve repair or replacement, replacement of the ascending aorta and aortic arch using the branch-first technique and frozen elephant trunk (FET). A thoracic endovascular aortic repair (TEVAR) is planned for the second stage.

\section{Surgical techniques}

The following describes our usual surgical techniques for FET cases, with additional comments specific to this case.

\section{Preparation}

The patient is positioned supine with adequate access to the groin. Intraoperative cerebral monitoring is performed by a combination of electroencephalogram bispectral index monitoring, cerebral oximetry (INVOS 3100; Somanetics Corp, Troy, Mich) and transcranial Doppler. Bilateral radial and lower limb pressure monitoring is performed routinely. Cerebrospinal fluid (CSF) drainage is not used routinely.

\section{Exposition}

The chest is opened by median sternotomy. For cardiopulmonary bypass $(\mathrm{CPB})$, arterial cannulation is performed in the ascending aorta and in this case, bicaval cannulation is used to enable concomitant mitral valve surgery. The left ventricle is vented via the right superior pulmonary vein. No guide-wire was used in this non-dissection case for the FET. Antegrade and retrograde blood cardioplegia techniques are utilised.

\section{Operation}

Our branch-first technique for aortic arch replacement has previously been described (1). The innominate and left common carotid branches are carefully exposed prior to institution of $\mathrm{CPB}$, whereas the left subclavian artery is exposed after institution of CPB. Initial cooling is slow and restricted to a minimum temperature of $32{ }^{\circ} \mathrm{C}$ to avoid ventricular fibrillation. A Dacron trifurcation graft with a perfusion side arm (TAPP graft, Vascutek Ltd, Renfrewshire, Scotland, UK) is selected and the branched limbs are trimmed to the appropriate length. First, the innominate artery is clamp isolated proximal to its bifurcation and then divided. The distal stump is anastomosed to the first limb of the trifurcation graft with 5/0 Prolene. De-airing manoeuvres are performed and then 
the clamps are rearranged to resume antegrade perfusion to the innominate artery from the side arm of the trifurcation graft. The innominate proximal stump is ligated with a silk suture. A similar process is repeated for the left carotid artery and left subclavian artery for the second and third limbs of the trifurcation graft. Antegrade cerebral perfusion is resumed in each case after careful de-airing through the trifurcation graft. Importantly, throughout the whole debranching process perfusion is not interrupted to the heart or distal organs.

The aorta is cross-clamped proximal to the arterial cannula and retrograde cardioplegia delivered. The sinotubular junction is transected and ostial antegrade cardioplegia is delivered. Antegrade and retrograde cardioplegia is repeated every 10 to 15 minutes. Core cooling is progressed to a target of $25^{\circ} \mathrm{C}$. Aortic and mitral valve replacement is completed while cooling.

On reaching target temperature, distal circulatory arrest is commenced and the aortic cannula and cross clamp is removed. The FET, either a Thoraflex hybrid graft (Vascutek Terumo, Inchinnan, Scotland UK) or E-Vita hybrid graft (Jotec GmbH, Hechingen, Germany), is deployed into the descending aorta. In this case, a Thoraflex hybrid graft is selected.

The collar of the device at the junction between the stent and the flexible graft is anastomosed to the native proximal descending aorta using a simple continuous 4/0 Prolene. De-airing manoeuvres are performed, and the graft is crossclamped. Distal perfusion is reinstated by antegrade inflow via the side arm of the graft. The main graft is trimmed to length and the proximal anastomosis to the sinotubular junction of the aortic root is performed. The common trunk of the trifurcation graft is delivered deep to the innominate vein and anastomosed to a hole formed in the new ascending aorta in an end-to-side fashion, without interrupting cerebral perfusion.

\section{Completion}

The heart is de-aired and cross-clamp removed. Routine atrial and ventricular temporary epicardial pacing wires are placed and CPB is weaned.

\section{Comments}

\section{Clinical results}

Thirty-seven patients have undergone aortic replacement using this branch-first technique with FET between October 2008 and June 2019. The median age was 65 years and the majority of patients were male (81\%).

$30 \%$ of cases were performed as urgent surgery with the remainder performed electively. Surgical indications included aortic aneurysm (73\%), acute aortic dissection (24\%) and chronic aortic dissection (22\%). Approximately half of all patients had prior cardiac surgical intervention.

There were no patients who had a CSF drain placed for their FET operation. Fourteen patients had concomitant proximal aortic procedures including aortic valve, root or valve-sparing root replacement. Median distal circulatory arrest, cross-clamp and perfusion times were 44, 145 and 278 minutes respectively.

Post-operatively, there were no cases of visceral malperfusion. There was one case of post-operative partial spinal ischemia with complete resolution. Two patients had pre-operative limb ischemia secondary to dissection however there were no new post-operative limb complications. Median ventilation time, intensive care unit and hospital length of stay were considerably shorter in the elective surgery subset (16 hours; 64 hours; 13 days respectively) compared with urgent surgery (107 hours; 176 hours; 20 days respectively).

There were three in-hospital mortalities all from the urgent surgery subset. One of these deaths occurred after an urgent staged procedure in the same admission. Three patients died out of hospital before their intended staged procedure.

Twenty-one patients had disease that could be treated completely by a single stage FET procedure. Twelve patients went on to have staged or completion distal aortic procedures with TEVAR $(n=7)$ or open thoracoabdominal aortic repair $(n=5)$. At the time of data collection, one patient was awaiting a staged procedure for a localised aortic dissection at the distal end of the FET.

\section{Advantages}

One of the advantages of the FET is that single-stage treatment is possible in cases where aortic pathology is limited in distal extent to the upper third of the descending thoracic aorta. In cases where distal aortic disease is not encompassed by the FET it provides a proximal landing zone for the second stage procedure, which may include TEVAR or thoracoabdominal repair. Additionally, the branch-first technique utilises a trifurcation graft for reimplantation of the arch vessels with the common trunk 
anastomosed to the proximal ascending aorta, and therefore provides generous landing zone in addition to that of the FET for subsequent TEVAR.

In the case of open surgery being required for a completion procedure, the FET eliminates the need to perform extensive dissection in the region of the distal arch, reducing the risk of recurrent laryngeal nerve and pulmonary artery injury. The extra buttressing provided by the FET increases the security of the anastomosis to the distal aorta especially in the case of extreme fragility such as aortic dissection.

In acute type $\mathrm{A}$ aortic dissection, the FET aids in expanding a collapsed true lumen. The stent may also cover proximal descending re-entry tears and thereby reduce the risk of ongoing pressurisation of the false lumen.

\section{Caveats}

There is a reported risk of spinal cord ischemia with FET grafts (2). This may be explained by prolonged circulatory arrest time, especially with moderate hypothermia and occlusion of intercostal arteries by the stent-graft (3). However, there has only been one case of spinal cord ischemia in our cohort to date and this arose in concert with generalised distal aortic hypoperfusion due to true lumen collapse despite the FET. This patient required urgent TEVAR distal extension with good restoration of distal true lumen flow and resolution of paraparesis. We hypothesise that this low rate of spinal cord ischemia is attributable to our branch-first technique which reduces the duration of distal circulatory arrest whilst also maximising collateral supply to the spinal cord by continuous antegrade cerebral perfusion.

In cases of aortic dissection there may be distal reentry tears, which if large enough, can cause retrograde pressurisation of the false lumen and compression of the FET. Consequently, there is a risk of obstruction to flow due to stent collapse in the FET. If this occurs, the FET stent may require further balloon dilatation and often placement of an extension covered stent graft, as mentioned previously.

\section{Acknowledgments}

None.

\section{Footnote}

Conflicts of Interest: The authors have no conflicts of interest to declare.

Open Access Statement: This is an Open Access article distributed in accordance with the Creative Commons Attribution-NonCommercial-NoDerivs 4.0 International License (CC BY-NC-ND 4.0), which permits the noncommercial replication and distribution of the article with the strict proviso that no changes or edits are made and the original work is properly cited (including links to both the formal publication through the relevant DOI and the license). See: https://creativecommons.org/licenses/by-nc-nd/4.0/.

\section{References}

1. Matalanis G, Perera NK, Galvin SD. Aortic arch replacement without circulatory arrest or deep hypothermia: the "branch-first" technique. J Thorac Cardiovasc Surg 2015;149:S76-82.

2. Shrestha M, Bachet J, Bavaria J, et al. Current status and recommendations for use of the frozen elephant trunk technique: a position paper by the Vascular Domain of EACTS. Eur J Cardiothorac Surg 2015;47:759-69.

3. Di Bartolomeo R, Murana G, Di Marco L, et al. Frozen versus conventional elephant trunk technique: application in clinical practice. Eur J Cardiothorac Surg 2017;51:i20-8.
Cite this article as: $\operatorname{Kim}$ M, Matalanis G. Aortic arch replacement using the branch-first and frozen elephant trunk techniques. Ann Cardiothorac Surg 2020;9(3):259-261. doi: 10.21037/acs.2020.03.01 


\section{University Library}

\section{- M M N E R VA A gateway to Melbourne's research publications}

Minerva Access is the Institutional Repository of The University of Melbourne

Author/s:

Kim, M;Matalanis, G

Title:

Aortic arch replacement using the branch-first and frozen elephant trunk techniques

Date:

2020-05-01

Citation:

Kim, M. \& Matalanis, G. (2020). Aortic arch replacement using the branch-first and frozen elephant trunk techniques. ANNALS OF CARDIOTHORACIC SURGERY, 9 (3), pp.259-261. https://doi.org/10.21037/acs.2020.03.01.

Persistent Link:

http://hdl.handle.net/11343/274458

License:

CC BY-NC-ND 